\title{
Associations between Physical Activity in Old Age and Dementia-Related Mortality: A Population-Based Cohort Study
}

\author{
Tor Atle Rosness $^{a} \quad$ Bjørn Heine Strand ${ }^{a, b} \quad$ Astrid Liv Mina Bergem ${ }^{d}$ \\ Knut Engedal ${ }^{c}$ Espen Bjertness ${ }^{a}$ \\ anstitute of Health and Society, University of Oslo, and ${ }^{b}$ Norwegian Institute of Public \\ Health, Oslo, 'Norwegian Centre for Ageing and Health, Vestfold Health Trust, Tønsberg, \\ and ${ }^{\mathrm{d}}$ Department of Geriatric Psychiatry, Akershus University Hospital, Lørenskog, Norway
}

Key Words

Dementia · Physical activity · Epidemiology · Geriatric medicine $\cdot$ Longitudinal studies

\begin{abstract}
Background: Findings from the literature vary in relation to whether physical activity brings about less cognitive decline in old age. The present study investigated self-reported levels of physical activity in old age and its association with the risk of dementia-related mortality. Methods: We included data from 31,086 subjects, between 65 and 80 years old, from the CONOR (Cohort of Norway) database. Cox regression analysis was used to estimate the risk of association. Results: Taking part in 'light' (not causing perspiration or panting) activities of $<3 \mathrm{~h}$ per week was associated with a decreased risk of dementia-related mortality, with a hazard ratio (HR) of 0.74 and a $95 \%$ confidence interval $(\mathrm{Cl})$ of $0.62-0.88$, and of $>3 \mathrm{~h}$ per week, with an $\mathrm{HR}$ of 0.61 and a $95 \% \mathrm{Cl}$ of $0.51-0.73$. When taking part in 'hard' (causing perspiration or panting) activities, a similar risk $(H R=0.56 ; 95 \% \mathrm{Cl} 0.43-0.72)$ was observed for $>3 \mathrm{~h}$ per week. Interestingly, the highest reduction in risk was seen for 'hard' activities of $<3 \mathrm{~h}$ per week $(\mathrm{HR}=0.50 ; 95 \% \mathrm{Cl} 0.41-0.61)$. Conclusion: Physical activity during leisure time in old age was associated with a lower risk of dementia-related mortality when compared to inactive individuals.

(C) 2014 S. Karger AG, Basel
\end{abstract}

\section{Introduction}

The epidemiological literature provides compelling evidence that physical activity is associated with a reduced risk of dementia late in life [1-5]. Physical activity during leisure time may further contribute to maintaining an apt cognitive ability throughout the process of 
ageing and is a possible way of preventing dementia [1,3]. Although being physically active in early midlife, and continuing with exercise in old age, could lower the risk of dementia, more longitudinal research is warranted since the effect may vary depending on characteristics such as apolipoprotein E status, overweight and gender [5]. Observational findings imply that regular physical activity is more effective in the delay of the onset of dementia compared to occasional bouts of exercise, but this is subject to dispute as some evidence appears to be of medium quality [2]. A dose-response relationship between physical activity and cognitive health has been established, but confounding issues require consideration, especially in genetically susceptible individuals [3, 4]. A systematic review assessing several health-related behaviours [e.g., smoking, body mass index (BMI), diet and nutrition] reported that physical activity during leisure time, even at a moderate level, resulted in protection against dementia [2]. Further, higher levels of physical activity in midlife appear to be independent of the individual's history of cerebrovascular disease when assessing the hazards of dementia occurring in an elderly population [1]. This posits multifactorial influence and a broader possible relationship between dementia and vascular risk factors to overall health, physical activity and functional status in the elderly, including genetic profiles [6].

Questions about physical activities during leisure time, implemented to assess the relationship of physical activity and dementia with a long-term follow-up, should include information about specific activities, such as walking, which is seen to be associated with less cognitive decline; however, the association is not solely dependent on the particular activity but also on the duration of the exercise session, i.e., hours per week as a decisive factor [7].

Physical activity in older individuals presupposes a good somatic status, unfaltering gait and balance as well as adequate motor skill performance. Poor physical function may precede symptoms of dementia not yet clinically manifested [8]. Elderly individuals who, when asked, answer to be in poor physical shape could reflect a past medical history prone to adverse lifestyle-related factors, increasing the risk of incipient dementia. An open question about perceived physical capability may reveal at-risk individuals who could benefit from preventive interventions [9].

The present study, including a large number of older adults, contributes, in a novel way, to the field of dementia research by its aim to longitudinally examine the association of selfreported levels of physical activity during leisure time with the risk of dementia-related mortality, in a prospective population-based register study.

\section{Patients and Methods}

The study population included 31,086 individuals from the Cohort of Norway (CONOR) database aged between 65 and 80 years at the time of examination. CONOR includes ten different epidemiological cohorts and is an established collaboration of population-based surveys in Norway [10]. Individuals were followed up for a maximum of 27 years (mean 10.3). The overall participation rate was $58 \%$ and includes a heterogeneous study population from different geographical areas in Norway with various age groups from a 10-year period (1994-2003). The data collection followed a standardized procedure, stipulated by the Norwegian Institute of Public Health and collaborating universities in Norway, where a letter of invitation, containing an information brochure, was mailed 2 weeks prior to the health examination. The participants returned a self-evaluated questionnaire by mail after completing the examination [10].

The attained educational level was coupled to participants using a unique personal identification number and the National Education Database, then divided into three groups: high (university degree/college and equivalents), medium (secondary qualifications) or low 
(public/elementary school), where low education corresponds to 7 years of schooling, middle education to 10 years and high education to $\geq 13$ years of schooling.

The health examination included a physical checkup, measuring the height, weight and blood pressure as well as drawing blood samples. Participants were asked to fill in a questionnaire assessing health-related variables pertaining to cardiovascular disease (CVD), diabetes, total cholesterol and smoking habits. Participants who reported suffering from diabetes (currently or previously) were categorized as diabetic. Participants were categorized as having a history of CVD, if they reported any incident of coronary heart disease, heart failure or angina pectoris. Participants' smoking habits were dichotomized into daily smoker or nondaily smoker, as proffered by the item that asked about such habits on the applied questionnaire. The BMI (weight in kilograms divided by height in meters squared) was divided conventionally into underweight (BMI <18.5), normal weight (BMI 18.5-24.9), overweight (BMI 25-29.9) and obesity (BMI >30). Hypertension was categorized according to the National Institute of Health guidelines as a systolic pressure of $\geq 160 \mathrm{~mm} \mathrm{Hg}$ and/or a diastolic pressure of $\geq 100 \mathrm{~mm} \mathrm{Hg}$ [11]. The total cholesterol level was grouped into four categories, suggested by the European Atherosclerosis Society: 5.20, 5.20-6.49, 6.50-7.79 and $\geq 7.80 \mathrm{mmol} / \mathrm{l}$ [12].

The question item on physical activity during leisure time was divided into categories of 'light' (not causing perspiration or panting) and 'hard' (causing perspiration or panting) physical activity, each with a four-graded answer related to hours per week of activity; 'none', ' $<1 \mathrm{~h}$ ', '1-2 h' and ' $\geq 3$ h'. Participants were asked to answer in accordance with their level of physical activity during the previous year and, when answering, to describe an average week of exercise. To avoid incomplete data sets of these two dichotomized groups, individuals who replied 'none' for the question of 'hard' activity were labelled missing if a reply did not exist for the light activity question (3.1\% of the participants). A missing reply for both questions was treated as missing data and excluded (9.4\%).

Dementia-related mortality was obtained through the Norwegian Cause of Death Registry (NCDR). Dementia was identified from death certificates, either as the underlying or accompanying cause, according to the ICD (10th revision) codes F00-F03 and G30.0-G30.9. Study members were followed from the date of the health examination until death, emigration or until January 1, 2012, whichever occurred first. The actual prevalence of dementia in Norway, according to death certificates, is believed to be well reflected by the prevalence of dementia in the NCDR $[13,14]$.

\section{Statistical Analysis}

To investigate the association between physical activity and old age with dementiarelated mortality, a set of Cox regression models were specified. Attained age was used as the time variable in the Cox regression, and competing events such as non-dementia-related mortalities or emigration were treated as censored. Cox proportional hazards models were used to compute hazard ratios (HRs) for dementia-related mortalities for subjects reporting on graded answers of physical activity. Participants defined as 'inactive' denoted the reference group. In order to mark a clear difference from the inactive group, the cutoff of being physically active was established as a threshold with a duration of $<3$ or $>3 \mathrm{~h}$ per week from the graded item on the questionnaire. First, the regression analyses were adjusted for age and gender. Then, a second model was introduced which further adjusted for self-reported health status and lifestyle and cardiovascular risk factors (history of CVD, diabetes, obesity, smoking and hypertension). In the final model, education was added. Schoenfeld residuals and graphical inspection were used to assess if the proportional hazards assumption was fulfilled. No violations of proportionality were observed. Estimates of age-adjusted dementia-related mortality rates, per 100,000 person-years, were performed using Poisson regression with person-years as offset. Stata version 13 was used for all analyses. 
Rosness et al.: Associations between Physical Activity in Old Age and DementiaRelated Mortality: A Population-Based Cohort Study

\section{Ethics}

All participants included in CONOR gave their written consent. The participants' names and personal ID numbers were omitted before data were made available for research purposes. The Norwegian Data Inspectorate and the Regional Committees for Medical Research Ethics approved the study. The study was conducted in accordance with the Declaration of Helsinki.

\section{Results}

Initially, our study population included 31,086 individuals. After removing missing cases without registered answers on the item concerning physical activity during leisure time ( $\mathrm{n}=$ 4,145 ), our study comprised data from 26,941 individuals, $65-80$ years old. The mean age at survey for all participants was 72.4 years (SD 3.6), with a mean age at death of 83.3 years (SD 4.7), while for individuals with dementia-related mortality, the mean age at survey was 73.8 years (SD 3.4), with a mean age at death of 83.8 years (SD 4.3; table 1). The participants were followed up for a maximum of 27 years (mean 10.3), during which the total number of deaths registered with a diagnosis of dementia stated in the death certificates was 1,601. When further stratified by age, comparing the rate of dementia-related deaths across various age groups, the results were as follows: $65-70$ years $(14.7 \%), 71-75$ years $(41.7 \%)$ and $76-80$ years (43.5\%) for dementia-related mortality rates (per 100,000 person-years; table 2). Individuals who reported any degree and duration of physical activity had a decreased risk of dementia-related mortality compared to individuals who reported to be inactive. Participants were asked to consider an average week of exercise, from the previous year, when answering. All levels of activity ranging from 'light' activity of $<3 \mathrm{~h}$ per week to 'hard' activity of $>3 \mathrm{~h}$ per week were significantly associated with a lower risk of dementia-related mortality compared to the inactive group (models 1-3, table 3). Those who performed 'light activity' (i.e., no activity causing perspiration or panting) of $<3$ and $>3 \mathrm{~h}$ per week had a significantly lower risk of dementia-related mortality than inactive individuals (HR $=0.74,95 \%$ CI 0.63 0.87 , and HR $=0.64,95 \%$ CI 0.54-0.76, respectively), after adjusting for age and gender (model 1, table 3). After adding education, history of CVD, hypertension, BMI, cholesterol, diabetes and smoking as covariates, the association remained robust and was not attenuated for $<3 \mathrm{~h}(\mathrm{HR}=0.74,95 \% \mathrm{CI} 0.62-0.88)$ and $>3 \mathrm{~h}$ of activities (HR $=0.61,95 \% \mathrm{CI} 0.51-0.73$; model 3, table 3). Interestingly, 'hard' activity lasting $<3 \mathrm{~h}$ per week showed the greatest reduction in the risk of dementia-related mortality (HR $=0.50,95 \%$ CI $0.41-0.60$ ) when adjusted for age and gender (model 1, table 3 ) and remained robust without attenuation after adjusting for all remaining covariates ( $\mathrm{HR}=0.50,95 \% \mathrm{CI} 0.41-0.61$; model 3 , table 3 ).

\section{Discussion}

We found a strong association between physical activity during leisure time and a lower risk of dementia-related mortality. 'Hard' activity of $<3 \mathrm{~h}$ per week showed the greatest reduction in the risk of dementia-related mortality $(\mathrm{HR}=0.50)$. The association remained robust after adjusting for several possible confounders including age, gender, education, smoking, history of CVD, BMI, total cholesterol, diabetes and hypertension, resulting in only marginal attenuation. A dose-response association between light activity of $<3 \mathrm{~h}$ per week $(\mathrm{HR}=0.74)$ and hard activity of $>3 \mathrm{~h}$ per week $(\mathrm{HR}=0.56)$, with a lower risk of dementiarelated mortality compared to inactive individuals, was further observed, and our findings present a tendency of a greater reduction in dementia-related mortality in the case of a longer 
Table 1. Background characteristics of the total study population (aged $65-80$ years) and of subjects with DRM

\begin{tabular}{l|l}
\hline Dement Geriatr Cogn Disord Extra 2014;4:410-418 \\
\hline DOI: $10.1159 / 000367938$ & $\begin{array}{l}\text { C 2014 S. Karger AG, Basel } \\
\text { www.karger.com/dee }\end{array}$ \\
\hline
\end{tabular}

Rosness et al.: Associations between Physical Activity in Old Age and DementiaRelated Mortality: A Population-Based Cohort Study

\begin{tabular}{|c|c|c|}
\hline & All participants & DRM \\
\hline Total & $31,086(100)$ & $1,601(100)$ \\
\hline Mean age at survey \pm SD, years & $72.4 \pm 3.6$ & $73.8 \pm 3.4$ \\
\hline Mean age at death $\pm S D$, years & $83.3 \pm 4.7$ & $83.8 \pm 4.3$ \\
\hline Women & $13,701(44)$ & 823 \\
\hline \multicolumn{3}{|l|}{ Education } \\
\hline Low & $13,755(44)$ & 802 \\
\hline Middle & $12,952(43)$ & 605 \\
\hline High & 4,208 (14) & 189 \\
\hline \multicolumn{3}{|l|}{ Diabetes } \\
\hline No & $28,443(93)$ & 1,425 \\
\hline Yes & $2,228(7)$ & 144 \\
\hline \multicolumn{3}{|l|}{ History of CVD } \\
\hline No & $23,488(76)$ & 1,199 \\
\hline Yes & $7,367(24)$ & 378 \\
\hline \multicolumn{3}{|l|}{ Daily smoking } \\
\hline No & $24,452(80)$ & 1,264 \\
\hline Yes & $6,126(20)$ & 291 \\
\hline \multicolumn{3}{|l|}{ BMI } \\
\hline$<18.5$ & $917(3)$ & 75 \\
\hline $18.5-24.9$ & $9,758(32)$ & 605 \\
\hline $25-29.9$ & $14,425(47)$ & 647 \\
\hline$>30$ & $5,680(18)$ & 241 \\
\hline \multicolumn{3}{|l|}{ Hypertension } \\
\hline No & $20,363(66)$ & 1,016 \\
\hline Yes & $10,606(34)$ & 566 \\
\hline \multicolumn{3}{|l|}{ Total cholesterol } \\
\hline$<5.20 \mathrm{mmol} / \mathrm{l}$ & $5,052(16)$ & 225 \\
\hline $5.20-6.49 \mathrm{mmol} / \mathrm{l}$ & $12,165(39)$ & 615 \\
\hline $6.50-7.79 \mathrm{mmol} / \mathrm{l}$ & $9,867(32)$ & 508 \\
\hline$>7.80 \mathrm{mmol} / \mathrm{l}$ & $3,941(13)$ & 249 \\
\hline \multicolumn{3}{|l|}{ Age groups } \\
\hline $65-69$ years & $8,590(28)$ & 236 \\
\hline $70-74$ years & $12,437(40)$ & 668 \\
\hline $75-80$ years & $10,059(32)$ & 697 \\
\hline DRM rate ${ }^{1}$ & & 309.4 \\
\hline
\end{tabular}

Figures in parentheses are percentages. DRM $=$ Dementia-related mortality.

${ }^{1}$ Per 100,000 person-years, adjusted for age in Poisson regression (the age of 70 years was set as the reference).

duration and a higher degree of physical activity, as others have observed previously [15]. Further, it seems that a threshold of $3 \mathrm{~h}$ per week of activity is not necessarily needed, but rather the fact of avoiding inactivity. However, these results need further confirmation.

Our findings suggest that being physically active per se could be a form of intervention to prevent dementia $[16,17]$. We advocate from our findings that individuals between the ages of 65 and 80 years will benefit more from some physical activity than from none, when considering the risk of dementia.

The overall low rate of dementia-related mortality (5.1\%) is probably related to participants that are deceased individuals and not diagnosed cases [13]. The time from the health examination survey until the registration of the demise lasted up to 27 years. We were not able to evaluate how cognitive symptoms might have changed during the course of these years or for how long participants suffered from dementia, nor whether physical activities 
Rosness et al.: Associations between Physical Activity in Old Age and DementiaRelated Mortality: A Population-Based Cohort Study

Table 2. Level and duration of physical activity for the total study population and for subjects with DRM

\begin{tabular}{|c|c|c|c|c|c|c|c|c|}
\hline & \multicolumn{2}{|c|}{$\begin{array}{l}\text { Study participants } \\
\text { (aged 65-80 years) }\end{array}$} & \multicolumn{2}{|c|}{ DRM, n } & \multicolumn{2}{|c|}{ DRM, \% } & \multicolumn{2}{|c|}{ DRM rate ${ }^{1}$} \\
\hline & men & women & men & women & men & women & men & women \\
\hline \multicolumn{9}{|l|}{ Physical activity } \\
\hline Inactive & 1,289 & 1,731 & 80 & 133 & 6.2 & 7.6 & 468.3 & 451.0 \\
\hline Light, <3 h per week & 3,727 & 4,071 & 192 & 243 & 5.1 & 5.9 & 364.4 & 335.1 \\
\hline Light, $>3$ h per week & 3,844 & 3,047 & 174 & 182 & 4.5 & 5.9 & 297.4 & 322.6 \\
\hline Hard, $<3$ h per week & 5,229 & 1,855 & 171 & 80 & 3.2 & 4.3 & 217.6 & 239.4 \\
\hline Hard, $>3$ h per week & 1,784 & 364 & 66 & 22 & 3.6 & 6.0 & 241.7 & 334.8 \\
\hline Total & 15,873 & 11,068 & 683 & 660 & 4.3 & 5.9 & 305.0 & 315.5 \\
\hline
\end{tabular}

DRM = Dementia-related mortality.

${ }^{1}$ Per 100,000 person-years, adjusted for age in Poisson regression (the age of 70 years was set as the reference).

Table 3. DRM HRs by level of physical activity

\begin{tabular}{|c|c|c|c|c|}
\hline & $\begin{array}{l}\text { Model } 0 \\
\text { (total = 26,941; } \\
\text { DRM =1,343) }\end{array}$ & $\begin{array}{l}\text { Model } 1 \\
\text { (total = 26,055; } \\
\text { DRM =1,296) }\end{array}$ & $\begin{array}{l}\text { Model } 2 \\
\text { (total = 26,055; } \\
\text { DRM =1,296) }\end{array}$ & $\begin{array}{l}\text { Model } 3 \\
\text { (total =26,055; } \\
\text { DRM =1,296) }\end{array}$ \\
\hline \multicolumn{5}{|l|}{ Physical activity } \\
\hline Inactive & 1.00 & 1.00 & 1.00 & 1.00 \\
\hline Light, $<3$ h per week & $0.74(0.63-0.87)$ & $0.75(0.63-0.89)$ & $0.74(0.62-0.87)$ & $0.74(0.62-0.88)$ \\
\hline Light, $>3$ h per week & $0.64(0.54-0.76)$ & $0.64(0.53-0.76)$ & $0.61(0.51-0.73)$ & $0.61(0.51-0.73)$ \\
\hline Hard, $<3$ h per week & $0.50(0.41-0.60)$ & $0.51(0.42-0.61)$ & $0.50(0.41-0.61)$ & $0.50(0.41-0.61)$ \\
\hline Hard, $>3$ h per week & $0.56(0.44-0.72)$ & $0.57(0.44-0.74)$ & $0.56(0.43-0.72)$ & $0.56(0.43-0.72)$ \\
\hline \multicolumn{5}{|l|}{ Overall test of } \\
\hline association & $<0.001$ & $<0.001$ & $<0.001$ & $<0.001$ \\
\hline
\end{tabular}

Figures in parentheses are 95\% CIs. DRM = Dementia-related mortality. Cox regression models, adjusted successively for gender, age, cardiovascular risk factors and educational level. Model 0: adjusted for age and gender (full sample). Models 1-3: reduced sample with non-missing values for all covariates in model 3. Model 1: adjusted for age and gender. Model 2: + adjustment for cardiovascular risk factors (hypertension, total cholesterol, BMI, self-reported diabetes and smoking). Model 3: + adjustment for education.

influenced other mental faculties, such as depression. In a well-known randomized trial, volunteers who reported memory problems and were thus considered to be at increased risk of dementia were included to assess the impact of a physical activity intervention program. After a 6-month program, only a modest improvement in cognition was seen [18].

Our participants might have been afflicted with clinically undetected memory problems prior to inclusion and possibly less inclined to be physically active. Inactivity was overall more prominent in females, as $4.7 \%$ of the men compared to $6.4 \%$ of the women were inactive. Furthermore, $7.6 \%$ of the women versus $6.2 \%$ of the men with dementia-related mortality were inactive. Concerning gender, studies have shown that women who report to be physically active at different points over their life course, especially when teenagers, have a lower likelihood of cognitive impairment later in life, and interventions should promote starting 


\begin{tabular}{l|l}
\hline DOI: 10.1159/000367938 & $\begin{array}{l}\text { (c) 2014 S. Karger AG, Basel } \\
\text { www.karger.com/dee }\end{array}$ \\
\hline
\end{tabular}

Rosness et al.: Associations between Physical Activity in Old Age and DementiaRelated Mortality: A Population-Based Cohort Study

with physical activity at an early age and recommend lifelong continuation [19]. Our findings support this notion.

It is a cumbersome undertaking to interpret long-term effects of physical activities and the subsequent occurrence of dementia. In accordance with our findings, since all participants who maintained some form of physical activity had a reduced risk of dementia-related mortality, the optimal level (or threshold) of physical activity to induce dementia protection presently remains unclear [20]. An important issue to contemplate in our observational study, when investigating physical activity and dementia, is whether less participation in leisure activities increases the risk of dementia per se or whether taking part in leisure activities, which entails a motivation for such activity, tends instead to subside during a preclinical phase of dementia. We were not able to entirely address this concern, as we did not know the moment when participants acquired a diagnosis of dementia. However, previous studies that showed leisure activities to be associated with a reduced risk of dementia also discovered a risk reduction when excluding subjects with possible preclinical dementia [21].

It is arduous to comprehend and beware of all possible mechanisms that could play a role in preventing dementia. Dichotomizing results into groups of individuals who either do or do not exercise is useful but also a simplification of the ageing process, and a threshold of $3 \mathrm{~h}$, although it allows for distinction from those that are inactive, is nonetheless arbitrary with regard to showing an impact on reducing dementia from being physically active. Through randomized interventions, and by applying neuroimaging tools, studies have shown that participation in physical activity increases the size of the prefrontal and hippocampal brain areas. Thus, physical activity may positively affect memory impairment since enlarged volumes of the prefrontal and hippocampal brain areas have been discerned in individuals who engage in more physical activity early in life [22].

Our findings concerning old age support previous studies that suggest exercise measured by self-reported items, specifying the frequency and degree of regular physical activity, serve as a valuable proxy when investigating the risk of dementia [23]. Our study is the first of its kind to include data concerning physical activity during leisure time from older adults with an outcome of dementia-related mortality. The strengths of our study include using a large collection of population-based data with a substantial follow-up time to longitudinally register patients that eventually developed dementia. Although the item on the CONOR questionnaire concerning physical activity is based on self-reporting, such brief and germane questions are valid when assessing the risk of dementia [10]. In our analysis, we adjusted for several known confounders and included different types of dementia, stipulated by the ICD (10th revision), since being physically active could predict a higher risk of different types of dementia [17].

Our study has some limitations. First, studying dementia-related mortality as a proxy for dementia illness could include death certificates with misclassifications of the underlying cause of death, such as other causes of death than those related to dementia or lacking a diagnosis of dementia, and non-deceased individuals with possible or probable dementia were not classified as cases in our study [24]. Second, we ascertained the diagnosis of dementia through the NCDR, which does not indicate the temporal aspect of how long a person has in fact suffered from dementia $[13,14]$. An underreporting of dementia on death certificates in the NCDR (low sensitivity) has been shown but the specificity of dementia on death certificates appears to be valid. In the case of a random underreporting in the NCDR, associations would be biased towards the null hypothesis due to loss of power [13]. Third, we relied upon self-reporting habits of physical activity during leisure time and answers could be prone to subjective experience and over- or underrepresentation of de facto intensity and duration of exercise. Fourth, participants were asked to imagine what an average week of exercise would be like from the previous year, and this could generate a representation bias due to inaccurate memory recall. Furthermore, injuries could have altered the participants' state of physical 
Rosness et al.: Associations between Physical Activity in Old Age and DementiaRelated Mortality: A Population-Based Cohort Study

capability and introduce a confounding bias. Lastly, the overall response rate of participants in CONOR was rather low (58\%) and could generate a selection bias, by healthier individuals being more likely to participate in health studies. However, due to our large study population, attrition was not considered likely in light of these possible influences [24].

In conclusion, our results, if the outcome is dementia-related mortality, support previous findings that being physically active in old age seems to lower the risk of dementia in a possible dose-response manner. Individuals performing activities to some extent $(<3$ or $>3 \mathrm{~h}$ per week) had a reduced risk of dementia-related mortality compared to inactive individuals, whereas those taking part in 'hard' activities for $<3 \mathrm{~h}$ had the most reduction in risk.

\section{Acknowledgement}

We would like to thank all contributing members of the GENIDEM research group.

\section{Disclosure Statement}

The authors declare no conflicts of interest.

\section{References}

1 Defina LF, Willis BL, Radford NB, Gao A, Leonard D, Haskell WL, Weiner MF, Berry JD: The association between midlife cardiorespiratory fitness levels and later-life dementia: a cohort study. Ann Intern Med 2013;158: 162-168.

2 Lee Y, Back JH, Kim J, Kim SH, Na DL, Cheong HK, Hong CH, Kim YG: Systematic review of health behavioral risks and cognitive health in older adults. Int Psychogeriatr 2010;22:174-187.

3 Rovio S, Kåreholt I, Helkala EL, Viitanen M, Winblad B, Tuomilehto J, Soininen H, Nissinen A, Kivipelto M: Leisure-time physical activity at midlife and the risk of dementia and Alzheimer's disease. Lancet Neurol 2005; 4:705-711.

4 Scarmeas N, Luchsinger JA, Schupf N, Brickman AM, Cosentino S, Tang MX, Stern Y: Physical activity, diet, and risk of Alzheimer disease. JAMA 2009;302:627-637.

5 Tolppanen AM, Solomon A, Kulmala J, Kåreholt I, Ngandu T, Rusanen M, Laatikainen T, Soininen H, Kivipelto M: Leisure-time physical activity from mid- to late life, body mass index, and risk of dementia. Alzheimers Dement 2014, Epub ahead of print.

6 Aarsland D, Sardahaee FS, Anderssen S, Ballard C: Is physical activity a potential preventive factor for vascular dementia? A systematic review. Aging Ment Health 2010;14:386-395.

7 Weuve J, Kang JH, Manson JE, Breteler MM, Ware JH, Grodstein F: Physical activity, including walking, and cognitive function in older women. JAMA 2004;292:1454-1461.

8 Wang L, Larson EB, Bowen JD, van Belle G: Performance-based physical function and future dementia in older people. Arch Intern Med 2006;166:1115-1120.

9 Kulmala J, Solomon A, Kåreholt I, Ngandu T, Rantanen T, Laatikainen T, Soininen H, Tuomilehto J, Kivipelto M: Association between mid- to late life physical fitness and dementia: evidence from the CAIDE study. J Intern Med 2014;276:296-307.

10 Naess O, Søgaard AJ, Arnesen E, Beckstrøm AC, Bjertness E, Engeland A, Hjort PF, Holmen J, Magnus P, Njølstad I, Tell GS, Vatten L, Vollset SE, Aamodt G: Cohort profile: cohort of Norway (CONOR). Int J Epidemiol 2008;37: 481-485.

11 NIH Heart, Lung and Blood Institute: What is high blood pressure? 2014. http://www.nhlbi.nih.gov/health/ health-topics/ topics/hbp/.

12 European Atherosclerosis Society: Cholesterol level cut-off scores. http://eurheartj.oxfordjournals.org/ content/9/5/571.

13 Barnes DE, Whitmer RA, Yaffe K: Physical activity and dementia: the need for prevention trials. Exerc Sport Sci Rev 2007;35:24-29.

14 Lautenschlager NT, Cox K, Kurz AF: Physical activity and mild cognitive impairment and Alzheimer's disease. Curr Neurol Neurosci Rep 2010;10:352-358.

15 Laurin D, Verreault R, Lindsay J, MacPherson K, Rockwood K: Physical activity and risk of cognitive impairment and dementia in elderly persons. Arch Neurol 2001;58:498-504. 
16 Lautenschlager NT, Cox KL, Flicker L, Foster JK, van Bockxmeer FM, Xiao J, Greenop KR, Almeida OP: Effect of physical activity on cognitive function in older adults at risk for Alzheimer disease: a randomized trial. JAMA 2008;300:1027-1037.

17 Hamer M, Chida Y: Physical activity and risk of neurodegenerative disease: a systematic review of prospective evidence. Psychol Med 2009;39:3-11.

18 Middleton LE, Barnes DE, Lui LY, Yaffe K: Physical activity over the life course and its association with cognitive performance and impairment in old age. J Am Geriatr Soc 2010;58:1322-1326.

19 Verghese J, Lipton RB, Katz MJ, Hall CB, Derby CA, Kuslansky G, Ambrose AF, Sliwinski M, Buschke H: Leisure activities and the risk of dementia in the elderly. N Engl J Med 2003;348:2508-2516.

20 Erickson KI, Weinstein AM, Lopez OL: Physical activity, brain plasticity, and Alzheimer's disease. Arch Med Res 2012;43:615-621.

21 Larson EB, Wang L, Bowen JD, McCormick WC, Teri L, Crane P, Kukull W: Exercise is associated with reduced risk for incident dementia among persons 65 years of age and older. Ann Intern Med 2006;144:73-81.

22 Strand BH, Langballe EM, Hjellvik V, Handal M, Næss O, Knudsen GP, Refsum H, Tambs K, Nafstad P, Schirmer H, Bergem AL, Selmer R, Engedal K, Magnus P, Bjertness E: Midlife vascular risk factors and their association with dementia deaths: results from a Norwegian prospective study followed up for 35 years. J Neurol Sci 2013; 324:124-130.

23 Hjellvik V, Engedal K, Handal M, Flaten TP, Langballe EM, Selmer R, et al: Dementia in the National Cause of Death Registry in Norway 1969-2010. Nor J Epidemiol 2012;22:8.

24 Bjertness E, Torvik A, Ince PG, Edwardson JA: Validation of Norwegian death certificates on dementia in residents of nursing homes. Epidemiology 1998;9:584-586. 
Filename: DEE367938

Article-No: 367938, Fig.: 0, Tab.: 3

$<$ Issueid $>003</$ Issueid $>$

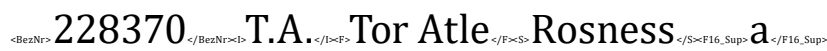

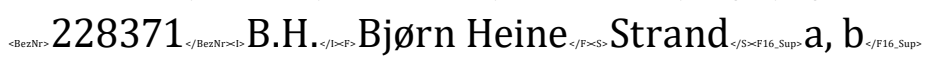

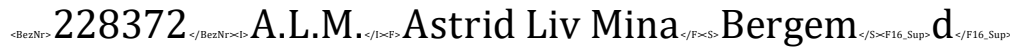

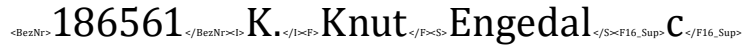

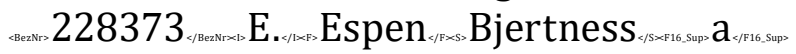

